\title{
Inclusion and exclusion criteria in research studies: definitions and why they matter
}

\author{
Cecilia Maria Patino 1,2,a, Juliana Carvalho Ferreira ${ }^{1,3, b}$
}

\section{PRACTICAL SCENARIO}

A cross-sectional multicenter study evaluated selfreported adherence to inhaled therapies among patients with COPD in Latin America. ${ }^{(1)}$ Inclusion and exclusion criteria for the study are shown in Chart 1 . The authors found that self-reported adherence was low in $20 \%$ of the patients, intermediate in $29 \%$, and high in $51 \%$; and that poor adherence was associated with more exacerbations in the past year, a lower smoking history, and a lower level of education. The authors concluded that suboptimal adherence to inhaled therapies among COPD patients was common and that interventions to improve adherence are warranted.

\section{BACKGROUND}

Establishing inclusion and exclusion criteria for study participants is a standard, required practice when designing high-quality research protocols. Inclusion criteria are defined as the key features of the target population that the investigators will use to answer their research question. ${ }^{(2)}$ Typical inclusion criteria include demographic, clinical, and geographic characteristics. In contrast, exclusion criteria are defined as features of the potential study participants who meet the inclusion criteria but present with additional characteristics that could interfere with the success of the study or increase their risk for an unfavorable outcome. Common exclusion criteria include characteristics of eligible individuals that make them highly likely to be lost to follow-up, miss scheduled appointments to collect data, provide inaccurate data, have comorbidities that could bias the results of the study, or increase their risk for adverse events (most relevant in studies testing interventions).

It is very important that investigators not only define the appropriate inclusion and exclusion criteria when designing a study but also evaluate how those decisions will impact the external validity of the results of the study. Common errors regarding inclusion and exclusion criteria include the following: using the same variable to define both inclusion and exclusion criteria (for example, in a study including only men, listing being a female as an exclusion criterion); selecting variables as inclusion criteria that are not related to answering the research question; and not describing key variables in the inclusion criteria that are needed to make a statement about the external validity of the study results.

\section{IMPACT OF THE INCLUSION AND EXCLUSION CRITERIA ON THE EXTERNAL VALIDITY OF THE STUDY}

In our example, the investigators described the inclusion criteria related to demographic characteristics (age $\geq$ 40 years of age and male or female gender), clinical characteristics (diagnosis of COPD, stable disease, outpatient, and current or former smoker); and exclusion criteria related to comorbidities that could bias the results (sleep apnea, other chronic respiratory diseases, and acute or chronic conditions that could limit the ability of the patient to participate in the study). On the basis of these inclusion and exclusion criteria, we can make a judgment regarding their impact on the external validity of the results. Making those judgments requires in-depth knowledge of the area of research, as well as of in what direction each criterion could affect the external validity of the study. As an example, the authors excluded patients with comorbidities, and it is therefore possible that the levels of nonadherence reported would not be generalizable to COPD patients with comorbidities, who most likely would show higher levels of nonadherence due to their more complex medication regimens.

Chart 1. Inclusion and exclusion criteria for a cross-sectional multicenter study of patients with COPD in Latin America. (1)

\begin{tabular}{ll}
\multicolumn{1}{c}{ Inclusion criteria } & \multicolumn{1}{c}{ Exclusion criteria } \\
- Adults $\geq 40$ years of age & - Diagnosis of sleep apnea or any other chronic respiratory \\
- Diagnosis of COPD at least for 1 year & disease \\
- At least one spirometry in the last year with a post- & - Any acute or chronic condition that would limit the ability \\
bronchodilator $\mathrm{FEV}_{1} / \mathrm{FVC}<0.70$ & of the patient to participate in the study \\
- Current or former smokers ( $>10$ pack-years) & - Refusal to give informed consent \\
- Stable disease (no recent exacerbation) & \\
\hline
\end{tabular}

\section{REFERENCES}

Montes de Oca M, Menezes A, Wehrmeister FC, Lopez Varela MV Casas A, Ugalde $L$, et al. Adherence to inhaled therapies of COPD patients from seven Latin American countries: The LASSYC study. PLoS One. 2017;12(11):e0186777. https://doi.org/10.1371/journal.

pone.0186777

2. Hulley SB, Cummings SR, Browner WS, Grady DG, Newman TB. Designing Clinical Research. 3rd ed, Philadelphia, PA: Lippincott Williams \& Wilkins; 2007

1. Methods in Epidemiologic, Clinical, and Operations Research-MECOR-program, American Thoracic Society/Asociación Latinoamericana del Tórax, Montevideo, Uruguay.

2. Department of Preventive Medicine, Keck School of Medicine, University of Southern California, Los Angeles, CA, USA

3. Divisão de Pneumologia, Instituto do Coração, Hospital das Clínicas, Faculdade de Medicina, Universidade de São Paulo, São Paulo (SP) Brasil.

a. (iD) http://orcid.org/0000-0001-5742-2157; b. (iD) http://orcid.org/0000-0001-6548-1384 